\title{
Transverse uncorrelated emittance diagnostic for magnetized electron beams
}

\author{
Fay Hannon ${ }^{1}$ and Mark Stefani ${ }^{2}$ \\ ${ }^{1}$ Thomas Jefferson National Accelerator Facility, Newport News, Virginia 23606, USA \\ ${ }^{2}$ Old Dominion University, Department of Electrical Engineering. Norfolk, Virginia 23529, USA
}

(Received 22 February 2019; published 23 October 2019)

\begin{abstract}
The study of magnetized electron beam has become a high priority for its use in ion beam cooling as part of electron ion colliders and the potential of easily forming flat beams for various applications. In this paper, a purpose-specific diagnostic is described with the intention of studying transverse magnetized beam properties. The device is a modification to the classic pepper-pot, used in this context to measure the uncorrelated components of transverse emittance in addition to the typical effective emittance. The limitations of traditional methods are discussed, and simulated demonstrations of the new technique shown.
\end{abstract}

DOI: 10.1103/PhysRevAccelBeams.22.102801

\section{INTRODUCTION}

Research at Thomas Jefferson National Accelerator Facility (JLab) has recently focused on the production of magnetized electron beams [1,2] for use within an electron cooler for the Jefferson Laboratory Electron Ion Collider (JLEIC) [3]. Magnetized electron bunches are generated when the source cathode is immersed within a magnetic field that has a field component normal to the emitting surface, typically provided by one or more solenoids. Due to the conservation of momentum, electrons gain angular momentum as they exit through the fringe of the magnetic field. This angular momentum significantly dominates transverse electron beam dynamics as shown in Fig. 1.

It is estimated that the cooling rate between a copropagating electron and ion beam in a solenoid channel could be improved by about two orders of magnitude if the electron bunch was not following Larmor rotations as it would with a typical electron beam [4,5]. With a magnetized beam, the angular momentum it has in free space can be removed precisely through the fringe fields of the cooling channel solenoid, such that it does not make large Larmor rotations, effectively reducing the emittance inside the solenoid.

The concept for the JLEIC cooler is to produce a bunched magnetized beam in the injector which is transported, without degradation, via a multipass energy recovery linac to a cooling solenoid [6,7]. The injector for the JLEIC cooler has a requirement for $3.2 \mathrm{nC}$ electron bunches at a continuous $43.3 \mathrm{MHz}$ repetition rate [8]. To produce

Published by the American Physical Society under the terms of the Creative Commons Attribution 4.0 International license. Further distribution of this work must maintain attribution to the author(s) and the published article's title, journal citation, and DOI. both the bunch charge and average current, DC electron guns are being considered due to being a more developed technology (over rf or SRF guns). A demonstration of both DC photocathode and thermionic cathode guns as a magnetized source are being investigated at JLab. A short diagnostic beam-line is used to characterize the electron beam as a function of magnetization, as shown in Fig. 2 [1].

The inherent angular momentum of magnetized beams manifests as a rotation and natural divergence in the transverse plane that complicates traditional diagnostic techniques, as described in the following sections. A further consideration is DC guns typically have a low electric field gradient at the cathode, and provide low overall energy gain $(<500 \mathrm{keV})$. This results in an operating regime where the beam dynamics of both space-charge forces in the electron bunch must be managed with the magnetized rotation. For highly magnetized bunches, the transverse beam size is typically large $(\sim 2 \mathrm{~cm}$ diameter compared with $\sim 0.5 \mathrm{~cm}$ from a nonmagnetized beam in this system) and the contribution of canonical angular momentum to the transverse emittance dominates transport.

Large transverse beams experience nonlinear off-axis fields in beamline elements such as magnets and $\mathrm{rf}$ structures. Any nonlinearity, either from space charge forces or electromagnetic fields can cause a degradation in the magnetization. Additionally, particle tracking tools, which are excellent predictors of performance for small, on-axis beams must also be bench-marked against measurement for this relatively unexplored regime. It is extremely important to be able to measure the uncorrelated emittance for the JLEIC electron cooler because this is the emittance present inside the cooling solenoid when copropagating with the ion beam. A diagnostic which quantifies the quality of magnetization and emittance is therefore of value both experimentally and as a simulation benchmarking tool. 

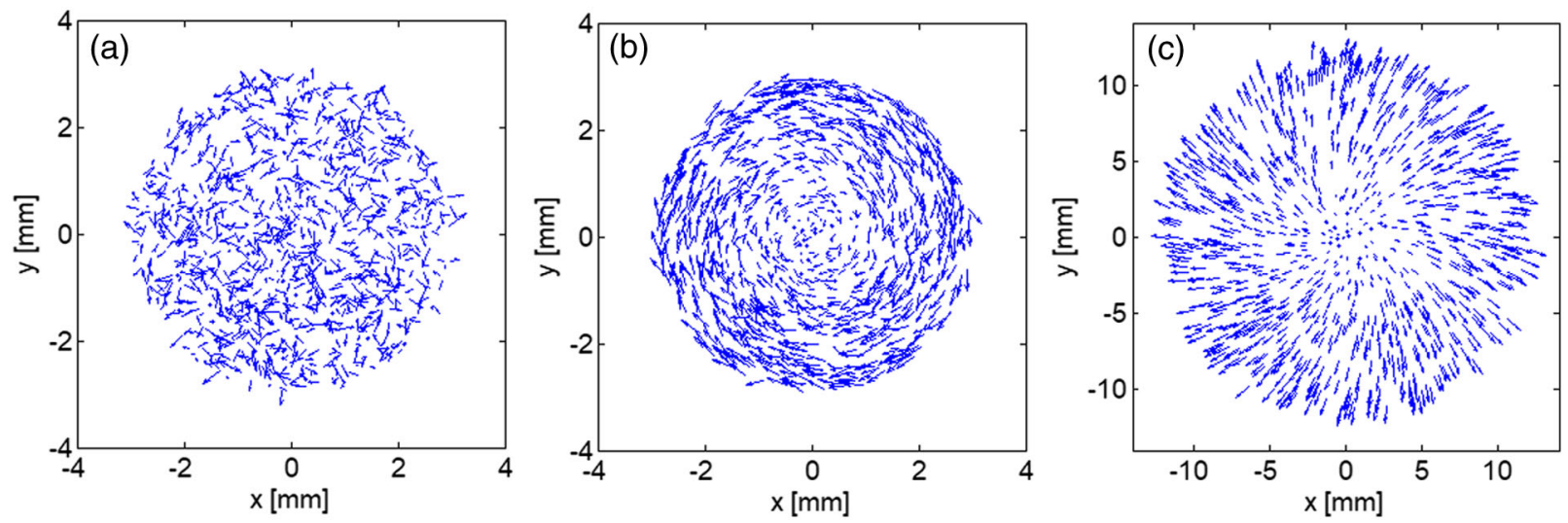

FIG. 1. Vector plots with arrows depicting individual particles in a beam, defined by transverse momentum in $\mathrm{x}$ and $\mathrm{y}$. (a) At the location of the cathode, inside the magnetic field, the random vectors are a result of the Maxwell Boltzmann thermal distribution of momenta. (b) Immediately downstream of the magnetic field at a beam waist, dominated by angular momentum. (c) Further downstream in free space where the beam is heavily divergent.

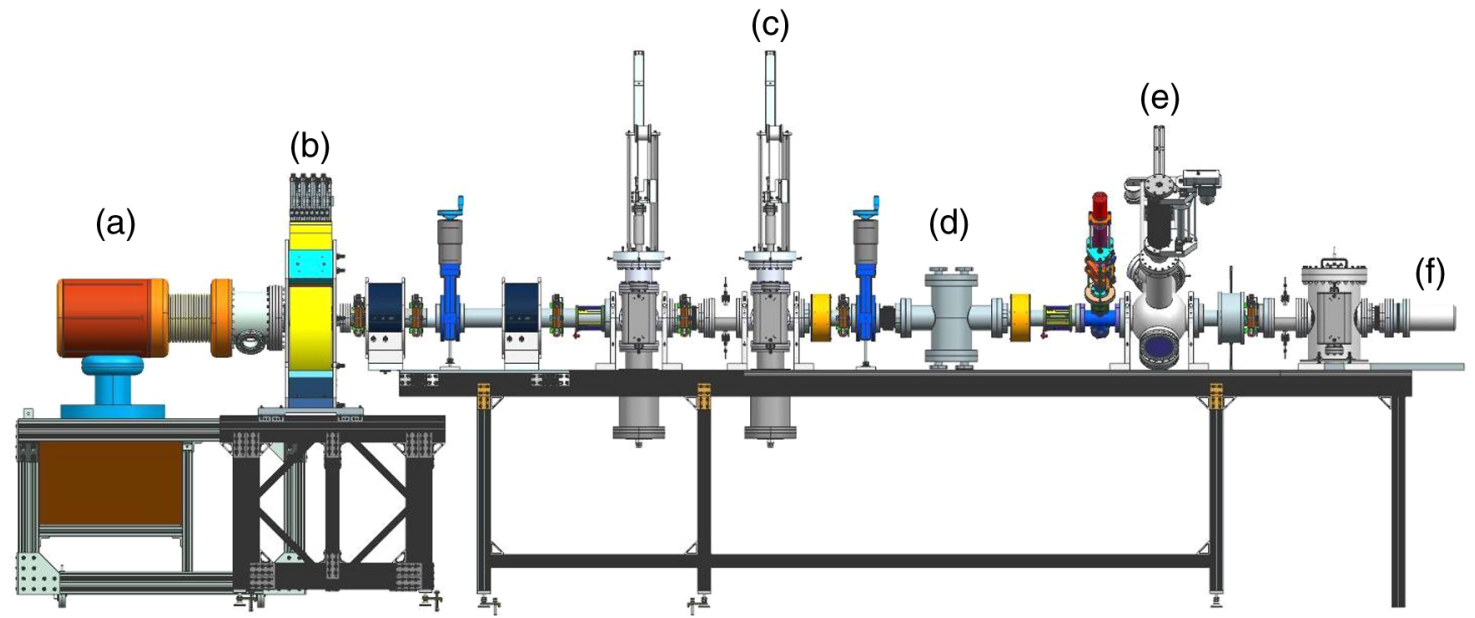

FIG. 2. Mechanical model of the test beamline. (a) thermionic gun (b) magnetizing solenoid (c) housing for 1D pepper-pot (d) deflecting cavity (e) housing for viewer screen (f) beam dump.

\section{EMITTANCE}

For a transversely cylindrically symmetric Gaussian beam with a sigma of $\sigma_{c}$, the average intrinsic canonical angular momentum, $\left\langle L_{i}\right\rangle$, is given by Eq. (1), where $e$ is the elementary charge of an electron and $B_{z}$ is the magnetic field strength at the cathode (perpendicular to the emitting plane).

$$
\left\langle L_{i}\right\rangle=e B_{z} \sigma_{c}^{2}
$$

The canonical transverse emittance, $\varepsilon_{m}$, that arises from the angular momentum is given by Eq. (2), where $m_{e}$ denotes the electron mass, and $c$ the speed of light (sometimes called drift, correlated or magnetized emittance). For the JLEIC cooler this quantity is set at $36 \mu \mathrm{m}$, thereby constraining the cathode emitting area to magnetic field ratio in accordance with Eq. (1). There is an obvious tradeoff between larger $\sigma_{c}$ and lower $B_{z}$; a bigger emitting radius is desirable to achieve the high bunch charge, whilst it is also a requirement that the magnetic field strength be uniform across the emitting area, which requires careful magnet design.

$$
\varepsilon_{m}=\frac{e B_{z} \sigma_{c}^{2}}{2 m_{e} c}
$$

In addition to the magnetized emittance, which has a linear correlation in the $x, \rho_{y}$ and $y, \rho_{x}$ planes (alternatively $r, \rho_{\phi}$ ), there is the typical uncorrelated emittance, $\varepsilon_{u}$, from thermal electron energy at the cathode, nonlinear fields and induced by space-charge forces within the bunch. For the JLEIC cooler there is a budget of $19 \mu \mathrm{m}$ for the uncorrelated emittance component. This is the emittance that will be 

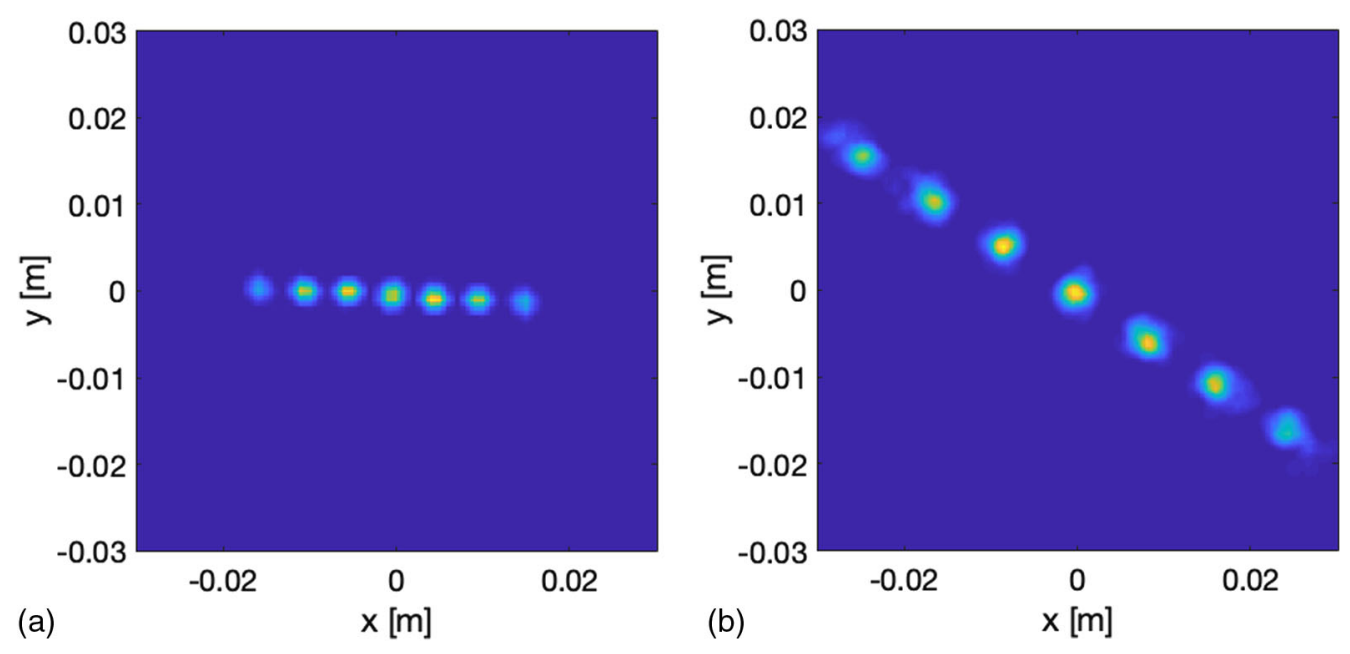

FIG. 3. Simulation (a) Initial beamlets at pepper-pot. (b) Final position of beamlets at viewer.

present inside the cooling solenoid when the correlated magnetized portion is removed as the beam passes through the fringe field. Minimizing this quantity improves cooling and decreases the chance of undesirable electron-ion recombination [9]. The total effective emittance, $\varepsilon$, is given by:

$$
\varepsilon^{2}=\varepsilon_{m}^{2}+\varepsilon_{u}^{2}
$$

\section{A. Emittance diagnostics}

Emittance measurement techniques at low energy $(<10 \mathrm{MeV})$ typically involve a insertable mask that allows a small portion of the beam to be transported (without space-charge forces) to a viewer, wire scanner or Faraday cup [10]. The emittance is then derived either statistically from the sampled beam or from an interpolated reconstruction of phase space. These masks are usually either slits or 2D pepper-pots. Single or multislits can be used to measure a 2D transverse phase space, while a pepper pot can measure both transverse planes simultaneously.

Consider a single slit diagnostic, where the beam is scanned over the aperture. A beamlet is passed through the slit and is incident on a viewer. In the case of magnetized beams, this beamlet is rotated and generally large at the viewer compared to nonmagnetized beams because of the angular momentum. For each transverse portion of the beam scanned, a beamlet is captured on the viewer. Each beamlet contains information about a slice of transverse phase space. The emittance can be calculated by statistically evaluating the first and second moments of position and angle, or a profile can be fitted to the divergent dimension of each beamlet and used to reconstruct phase space $[11,12]$. With a reconstruction, one can determine what measurement is most important, such as fractional or core emittance. Furthermore, reconstructed phase space images can be visually compared with simulation as a validation tool.
Multislits and 2D pepper-pots can be problematic with highly divergent beams as beamlets can overlap and one has to choose a method of chopping the image to assign a portion to each slit or hole for calculation. If the divergence is nonuniform across the radius of the beam or asymmetric, though not impossible, the image division for analysis becomes more subjective. Magnetized beam also causes a rotation of the beamlet as seen on the viewer, see Fig. 3(b) as an example. If multislits or pepper-pots are designed with spacing such that beamlets do not overlap at the viewer, the limited sampling of the beam leads to an underestimate of the emittance. They must then be used in conjunction with beam scanning over the mask to accurately cover phase space.

At JLab, as part of a magnetized beam test from a thermionic gun, a 1D pepper-pot was designed as a longitudinal bunch profile diagnostic. The 1D pepper-pot has distinct advantages for longitudinal measurements as described in [13]. For magnetized beams the 1D pepper-pot can also be used to provide both transverse phase space images when combined with beam scanning in both the horizontal and vertical plane. Figure 3 shows the typical behavior of beamlets as simulated from a 1D pepper-pot. Figure 3(a) displays some slight rotation because the center of the bunch is at the mask location and the head, which is downstream is already diverging.

This 1D pepper-pot has been designed with an array of 15 horizontal holes spaced such that beamlets will not overlap for a large operating region, as depicted in Fig. 4. For vertical emittance measurements, the beam is scanned over the pepper-pot vertically using carefully calibrated steering magnets. Shown in Fig. 5 is a simulation of a beam image on the viewer, combined for 5 vertical positions. The analysis to reproduce phase space (or statistically calculate emittance) requires that each beamlet be assessed individually. With this design, one can mask unwanted beamlets without losing information. For each beamlet the first and second moments in the $x$ and $y$ plane of the viewer are evaluated and an intensity profile taken. Over many vertical 


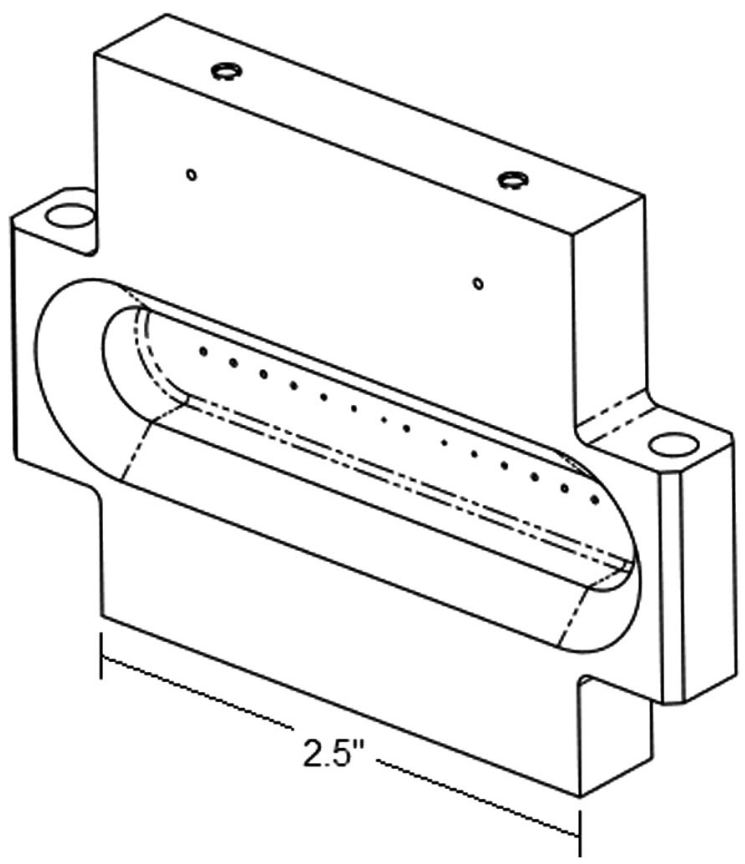

FIG. 4. Mechanical drawing of the 1D pepper pot.

measurements, one can reconstruct vertical phase space at the location of the diagnostic. This yields the total effective vertical emittance $\varepsilon_{y}$.

By changing perspective of the image shown in Fig. 5, one can view the line of holes in the average rotated plane to gain insight into the uncorrelated emittance. By calculating the average rotation and removing this from the image, Fig. 6 is generated. The procedure of dividing each beamlet and calculating profiles and moments is repeated. Now, the total vertical uncorrelated emittance is revealed, $\varepsilon_{u, y}$. The horizontal emittances, $\varepsilon_{x}$ and $\varepsilon_{u, x}$

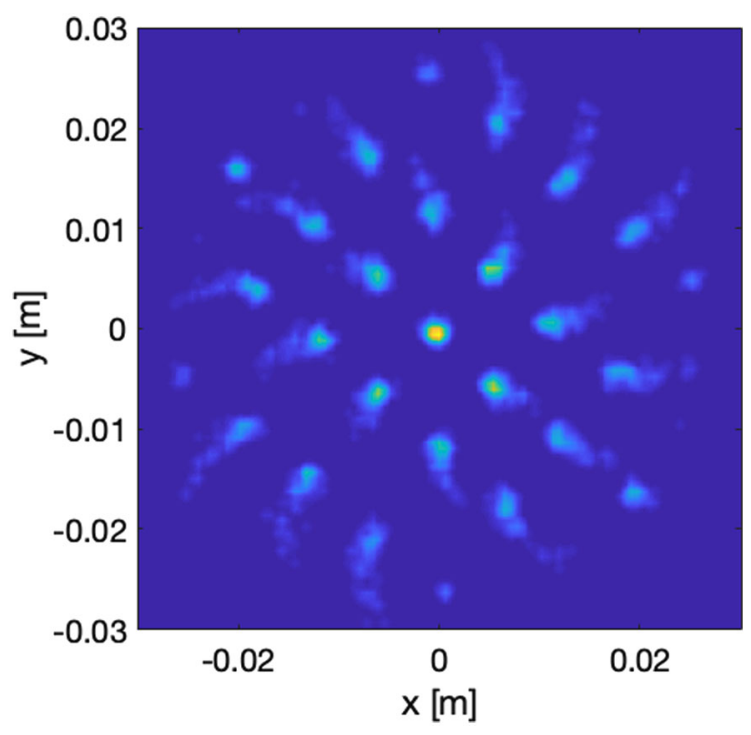

FIG. 5. Simulated viewer image of 5 superimposed vertical measurements.

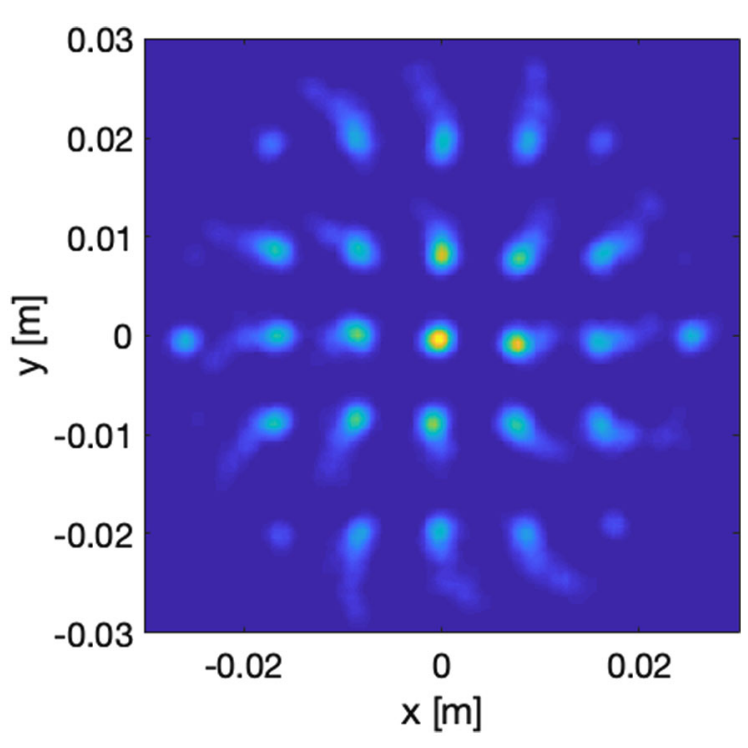

FIG. 6. Simulated viewer image of 5 superimposed vertical measurements, rotated to remove the average angular momentum.

require additional horizontal beam scanning to create more data points between each horizontal hole.

\section{B. Angular momentum}

As touched upon in the previous section, the average angular momentum, $\langle L\rangle$, can be calculated by fitting a line to a row of beamlet images on the viewer and using Eq. (4). $\sigma_{0}$ is the rms beam size at the mask, $\sigma_{v}$ the rms beam size at the viewer, $D$ the distance between mask and viewer, and $p_{z}$ longitudinal momentum.

$$
\langle L\rangle=p_{z} \frac{\sigma_{0} \sigma_{v} \sin (\theta)}{D}
$$

It should be noted that this is not necessarily equal to the intrinsic angular momentum, $\left\langle L_{i}\right\rangle$ from the cathode as external forces or those from within the bunch cause additional radial momentum components. For $\langle L\rangle=\left\langle L_{i}\right\rangle$ the beam must be at a waist at the mask so that transport between the mask and screen is purely defined by the angular momentum. Rather than average angular momentum, it may be of interest to fit a function to $L(x)$. For example the central horizontal slice in Fig. 6 shows some curvature at the extremities that is still present after the average rotation is removed, and this may be a good indicator of how well the magnetization is preserved.

\section{SIMULATION}

In this section the beamline described in Fig. 2 has been simulated using the particle tracking code GPT [14]. A $125 \mathrm{kV}$ thermionic gun was modeled, including the magnetizing solenoid and focusing elements. The cathode emitting area and magnetic field strength were set to give a 


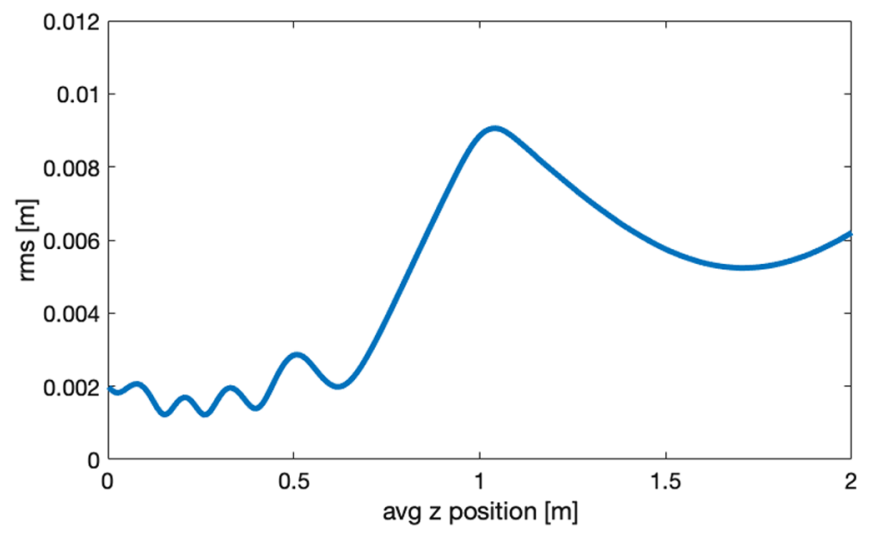

FIG. 7. Transverse rms beam size evolution from cathode to 1D pepper-pot.

correlated magnetized emittance of $36 \mu \mathrm{m}$ to match the JLEIC cooler specification, but with a reduced bunch charge of $135 \mathrm{pC}$ (limited by the cathode). The estimated thermal emittance contribution from the cathode was included in the simulation, and a focusing solenoid was used at $1 \mathrm{~m}$. The electron bunch was tracked to the location of the 1D pepper-pot at $1.98 \mathrm{~m}$ from the cathode. Figure 7 shows the transverse beam size evolution along the beamline. The beam in this simulation is axially symmetric. Figure 8 depicts the normalized rms effective emittance and the uncorrelated component. The rms uncorrelated emittance is calculated by fitting a least-square method line of best fit, with gradient $m$ to the $\left(y, \rho_{x}\right)$ phase space and calculating nonrotated momenta: $\rho_{y}=\rho_{y 0}-m x$ where $\rho_{y 0}$ is the original momentum.

To demonstrate how this diagnostic would be used in practice, a virtual experiment in simulation was performed with 100k particles that were tracked in GPT utilizing a space charge algorithm to the location of the pepper-pot.
Beyond the location of the pepper-pot, the particle distribution is manipulated analytically. First the 15 hole mask is applied to the distribution, adjusting the hole size to $0.8 \mathrm{~mm}$ to allow a statistically meaningful number of particles to pass through the holes. This is compared to the actual minimum hole size of $0.3 \mathrm{~mm}$. This allows $\sim 1 \%$ of the particles to pass through. The beamlets from individual holes were then tracked to the location of the viewer assuming no space charge. A 2D histogram was used to bin particles at the viewer to emulate the intensity of pixels of a camera looking at the viewer. The bin size was chosen to $0.1 \mathrm{~mm} \times 0.1 \mathrm{~mm}$ (comparable with typical cameras at JLab). Smooth horizontal and vertical profiles are fit to each histogram, using a SuperGaussian function for reconstruction and again after the average rotation is removed for the uncorrelated emittance. This is repeated for 21 vertical slices of the bunch.

The phase space for the effective emittance and the uncorrelated emittance as simulated directly from GPT are compared with the reconstruction from the virtual experiment. These are shown in Figs. 9 and 10 respectively.

The calculated effective emittance from the reconstruction is $36.24 \mu \mathrm{m}$, compared to $36.08 \mu \mathrm{m}$ directly from the simulation. The calculated uncorrelated emittance from the reconstruction is $5.03 \mu \mathrm{m}$, compared to $4.91 \mu \mathrm{m}$ directly from the simulation. Even with only $\sim 1 \%$ of the beam being sampled for use in reconstruction, the effective emittance is within $0.5 \%$ accuracy. Visual comparison also shows good agreement. The uncorrelated emittance is within $2.5 \%$ accuracy. There is a larger discrepancy between the simulated and reconstructed uncorrelated emittance due to statistical errors with a smaller divergence (fewer pixels), and in part to the tails on the beamlets from the nonlinear rotation present on the transverse edge of the bunch. The phase space reconstruction was a simple

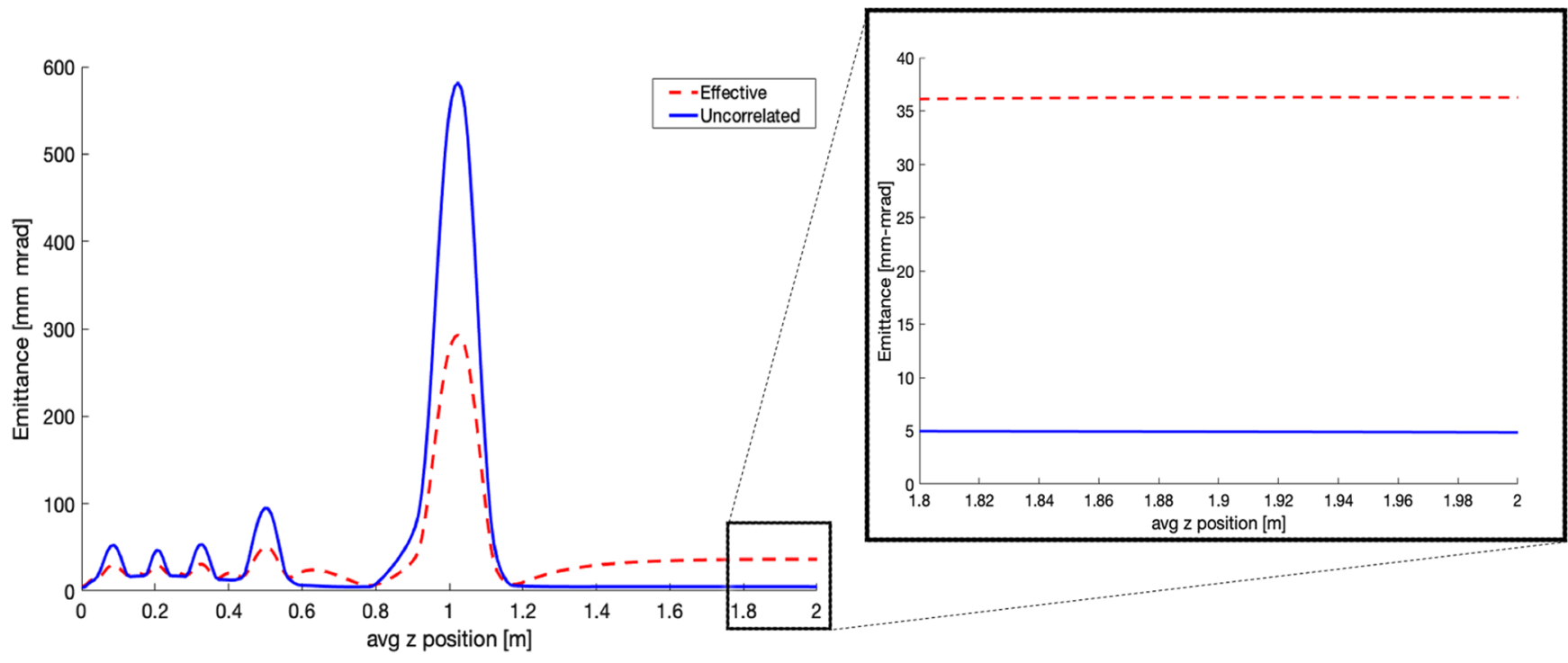

FIG. 8. The evolution of effective rms emittance (red dashed) and uncorrelated rms emittance (blue) from cathode to 1D pepper-pot. 


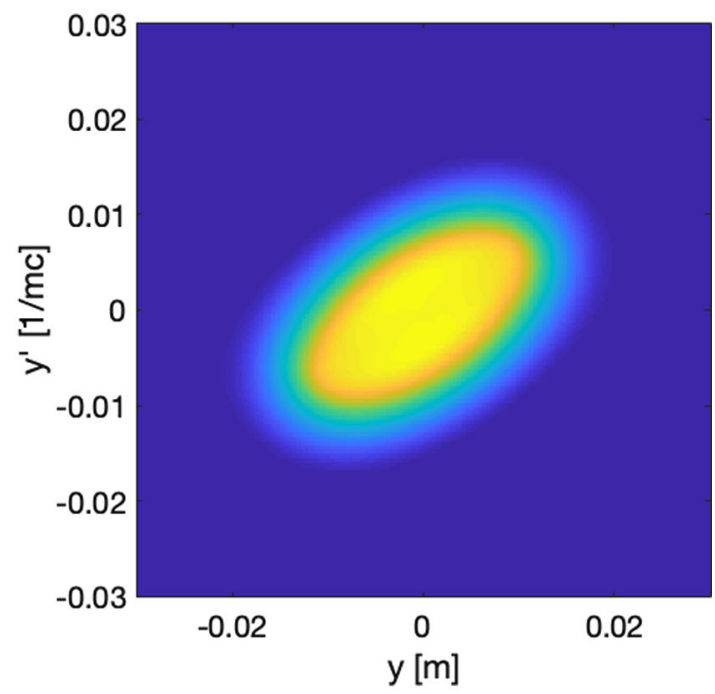

Simulated.

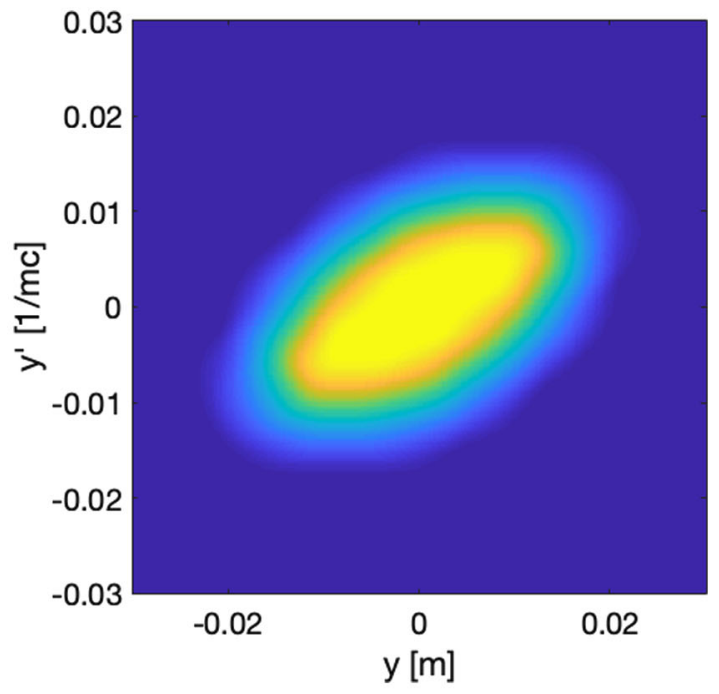

Reconstructed from 21 slices.

FIG. 9. Vertical effective phase space, (left) simulated, (right) reconstructed.

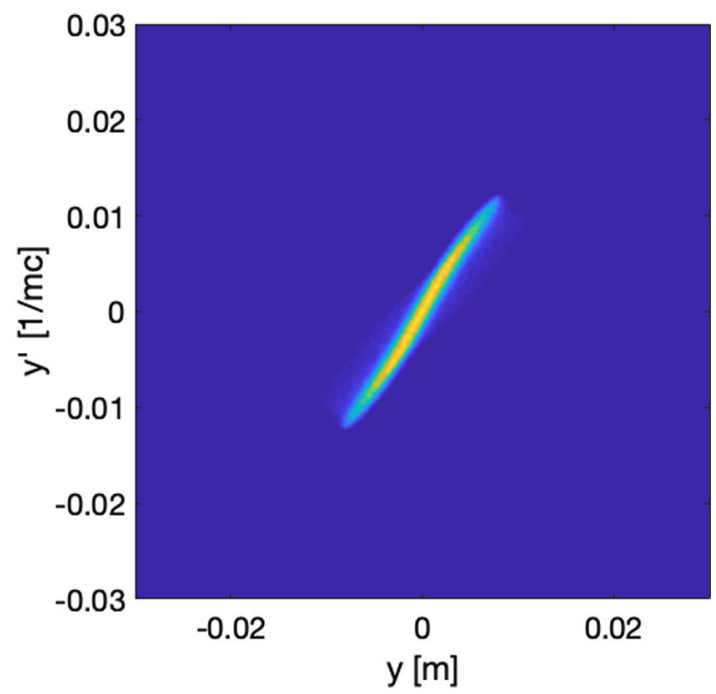

Simulated.

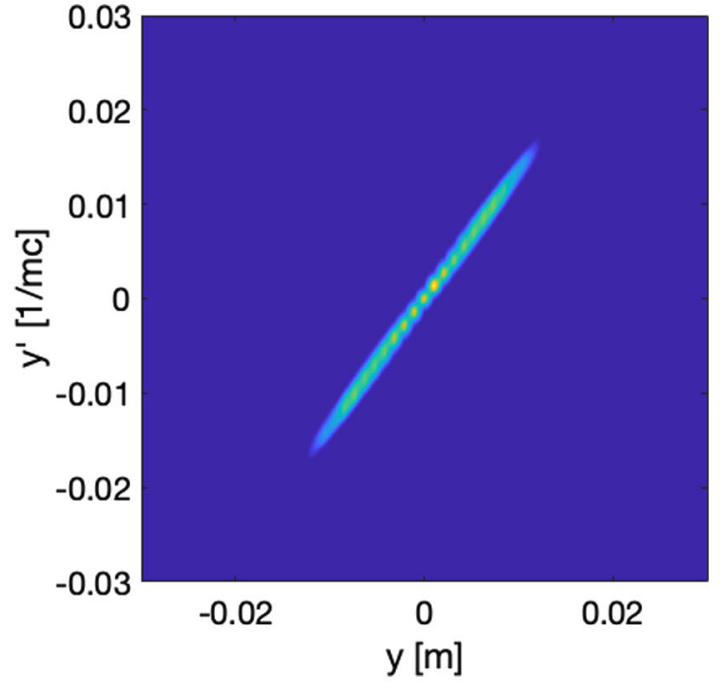

Reconstructed from 21 slices.

FIG. 10. Vertical uncorrelated phase space, (left) simulated, (right) reconstructed.

interpolation between beamlets. Therefore, the nonlinear tails of the beamlets on the rotated image increase the measured emittance giving an overestimation. Improvements in accuracy could be made by tracking more particles, additional scanning of the beam over the pepper-pot or by the use of different reconstruction algorithms as found in tomography, for example.

\section{CONCLUSION}

The simulation results demonstrate that a 1D pepper pot is a new diagnostic that can be utilized to reconstruct and visualize the uncorrelated transverse phase space component of magnetized beams in addition to the traditionally viewed effective phase space. With beam scanning horizontally and vertically, both transverse planes can uniquely be generated with this diagnostic.

There is a further advantage of the $1 \mathrm{D}$ pepper pot over a slit diagnostic when simply looking at a rotated image downstream, in that the magnetization of the beam in the transverse $\left(r, \rho_{\phi}\right)$ plane can be assessed also.

For the success of the JLEIC cooling scheme it is critical to know that the magnetization of the beam has been preserved from the cathode and that the uncorrelated component of transverse phase space is within specification. In the low energy $(<10 \mathrm{MeV})$ region, this diagnostic technique yields quantitative information on both of these parameters. 


\section{ACKNOWLEDGMENTS}

This manuscript has been authored by Jefferson Science Associates, LLC under U.S. DOE Contract No. DE-AC0506OR23177. The United States Government retains and the publisher, by accepting the article for publication, acknowledges that the United States Government retains a nonexclusive, paid-up, irrevocable, world-wide license to publish or reproduce the published form of this manuscript, or allow others to do so, for United States Government purposes.

[1] M. A. Mamun et al., Production of magnetized electron beam from a dc high voltage photogun, Proceedings of the 9th International Particle Accelerator Conference (IPAC2018), Vancouver, BC, Canada (JACoW Publishing, Geneva, Switzerland, 2018), https://doi.org/10.18429/ JACoW-IPAC2018-THPMK108.

[2] S. A. K. Wijethunga, M. A. Mamun, F. E. Hannon, G. A. Krafft, J. Benesch, R. Suleiman, M. Poelker, and J. R. Delayen, Simulation study of the magnetized electron beam, Proceedings of the 9th International Particle Accelerator Conference (IPAC2018), Vancouver, BC, Canada (JACoW Publishing, Geneva, Switzerland, 2018), https:// doi.org/10.18429/JACoW-IPAC2018-THPAK071.

[3] G. H. Wei, V. S. Morozov, F. Lin, Y. Zhang, Y. Cai, and Y. M. Nosochkov, Status of the JLEIC ion collider ring design, Proceedings of the 9th International Particle Accelerator Conference (IPAC2018), Vancouver, BC, Canada (JACoW Publishing, Geneva, Switzerland, 2018), https:// doi.org/10.18429/JACoW-IPAC2018-MOPML002.

[4] Ya. Derbenev, Adapting optics for high-energy electron cooling, University of Michigan Report No. UM-HE-9804, 1998.

[5] A. S. Artamonov and I. S. Derbenev, Positron cooling in a magnetized electron beam, Zh. Eksp. Teor. Fiz. 94, 15 (1988) [Sov. Phys. JETP 67, 5 (1988)].

[6] H. Zhang, S. V. Benson, Y. S. Derbenev, Y. Roblin, and Y. Zhang, Multi-stage electron cooling scheme for JLEIC, in
Proceedings of the 9th International Particle Accelerator Conference (IPAC2018), Vancouver, BC, Canada (JACoW Publishing, Geneva, Switzerland, 2018), https:// doi.org/10.18429/JACoW-IPAC2018-MOPML006.

[7] S. Benson et al., Development of bunched beam electron cooler for the Jefferson Lab Electron-Ion Collider, in Proceedings of the 9th International Particle Accelerator Conference (IPAC2018), Vancouver, BC, Canada (JACoW Publishing, Geneva, Switzerland, 2018), https:// doi.org/10.18429/JACoW-IPAC2018-MOPMK015.

[8] F.E. Hannon, Design of a high charge, low energy, magnetized electron injector, in Proceedings of the 9th International Particle Accelerator Conference (IPAC2018), Vancouver, BC, Canada (JACoW Publishing, Geneva, Switzerland, 2018), https://doi.org/10.18429/ JACoW-IPAC2018-THPMK107.

[9] A. Burov, Ya. Derbenev, S. Nagaitsev, and A. Shemiakin, Optical principles of beam transport for relativistic electron cooling, Phys. Rev. Accel. Beams 3, 094002 (2000).

[10] M. Zhang, Emittance formula for slits and pepper-pot measurement, Technical Report FNAL-TM-1988, Fermi National Accelerator Lab., Batavia, IL (United States), 1996, https://doi.org/10.2172/395453.

[11] M. E. Dolinska and N. L. Doroshko, Pepper-pot diagnostic method to define emittance and Twiss parameters on low energies accelerators, Problems of Atomic Science and Technology, 2, 107 (2002).

[12] S. G. Anderson, J. B. Rosenzweig, G. P. LeSage, and J. K. Crane, Space-charge effects in high brightness electron beam emittance measurments, Phys. Rev. ST Accel. Beams 5, 014201 (2002).

[13] M. Stefani and F. Hannon, Longitudinal bunch profile diagnostic for magnetized electron beams, Phys. Review Accel. Beams (to be published).

[14] S. B. van der Geer and M. J. de Loos, Applications of the general particle tracer code, Proceedings of the 1997 Particle Accelerator Conference (Cat. No. 97CH36167) (1997), Vol. 2, pp. 2577-2579, https://doi.org/10.1109/ PAC.1997.751279 